\title{
STABILITY OF WAFER LEVEL VACUUM ENCAPSULATED SINGLE-CRYSTAL SiLICON RESONATORS
}

\author{
Ville Kaajakari ${ }^{1}$, Jyrki Kiihamäki ${ }^{1}$, Aarne Oja ${ }^{1}$, Heikki Seppä ${ }^{1}$, Sami Pietikäinen ${ }^{2}$, Ville Kokkala ${ }^{2}$, and Heikki Kuisma ${ }^{2}$ \\ ${ }^{1}$ VTT Information Technolog, P.O.B. 1207, FIN 02044 VTT, Finland \\ ${ }^{2}$ VTI Technologies Oy, P.O.B. 27, FIN 01621 Vantaa, Finland \\ E-mail: ville.kaajakari@vtt.fi
}

\begin{abstract}
Stability of wafer level vacuum encapsulated micromechanical resonators is characterized. The resonators are etched of silicon-on-insulator (SOI) wafers using deep reactive ion etching (DRIE) and encapsulated with anodic bonding. Bulk acoustic wave (BAW) resonator show drift better than 0.1 $\mathrm{ppm} / \mathrm{month}$ demonstrating that the stability requirements for a reference oscillator can be met with MEMS. The drift of flexural resonators range from $4 \mathrm{ppm} / \mathrm{month}$ to over $500 \mathrm{ppm} / \mathrm{month}$ depending on resonator anchoring. The large drift exhibited by some flexural resonator types is attributed to packaging related stresses.
\end{abstract}

\section{INTRODUCTION}

Stable frequency references are required in wide range of electronic equipment. The typical stability requirements range from a few ppm a year for low cost clocks to less than a ppm for reference oscillators [1]. Conventionally, quartz crystals are used for the frequency reference as the good material stability of quartz allows low aging rates that meet the strict frequency precision requirements [2]. Furthermore, the quartz oscillators can provide excellent spectral purity (low phase noise), which is utilized in receivers for improved sensitivity and frequency selectivity [3]. A major disadvantage of quartz crystals is their large size which makes the frequency reference a significant space consumer in the todays highly integrated electronics.

Micromechanical resonators have been researched as a potential alternative to the size consuming quartz crystals. Clamped-clamped beam resonators, also referred to as the bridge resonators, have been used to demonstrate oscillator with a noise floor of $-110 \mathrm{dBc} / \mathrm{Hz}$ [4]. In addition, temperature compensation of clamped-clamped beam resonator has also been demonstrated [5]. Unfortunately, the power handling capacity of clamped-clamped resonators limit the achievable phase noise [6], [7]. Orders-of-magnitude larger power handling capacity has been obtained with a bulk acoustic mode (BAW) silicon resonators leading to noise floor of $-150 \mathrm{dBc} / \mathrm{Hz}$ at $13 \mathrm{MHz}$ meeting the typical phase noise requirements for wireless communication [8], [9]. Recently, comparable noise performance has been obtained with a polycrystalline silicon disk resonator and integrated electronics [10].

While the short term stability (phase noise) has received significant attention, very little published information exists on the long term frequency stability of the microresonators. Unpackaged torsional mode resonators show drift of more than $100 \mathrm{ppm} /$ day [11]. Unpackaged BAW resonators demonstrate stability better than $50 \mathrm{ppm} /$ week [12]. The superior stability performance of the BAW resonators may be attributed to their larger volume-to-surface ratio. Water adsorbing is believed to be the main cause of drift in unpackaged components. Surface treatment of the unpackaged torsional resonators reduces the drift to less than $100 \mathrm{ppm} /$ week [11]. Ceramic vacuum encapsulated BAW resonators have been demonstrated yield drift less than $1 \mathrm{ppm}$ in 40 days [12].

This paper reports on stability of silicon resonators packaged with a commercial wafer level vacuum encapsulation process [13]. Different microresonators packaged in the same process are compared and reference quality stability performance is demonstrated.

\section{FABRICATION AND PACKAGING}

Figure 1(a) and 1(b) show images of vacuum encapsulated resonators (size: $1 \mathrm{~mm}^{3}$ ) fabricated with the process shown in Figure 2. The devices were made by deep reactive ion etching of silicon-on-insulator (SOI) wafers. The space between the cap and resonator was done by recessing the SOI wafer with thermal oxidation. The resonating structures were released by etching of the sacrificial oxide with hydrofluoric acid followed by critical point drying. After the release, the devices were vacuum encapsulated by anodic bonding a glass wafer with silicon feed throughs to hermetically seal the resonators a wafer level encapsulation process used in the low cost volume production of MEMS accelerometers, gyroscopes, and pressure sensors [13].

Having the feed throughs in the glass cover greatly simplifies the fabrication process as no electrically isolated feed throughs are needed on the silicon wafer. The structure is also very compact as the electrical contacts are on top of the resonator. The pressure inside the packages is estimated to be $1 \mathrm{mBar}$. The resistances of the feed through and the feed though to wafer contact were $10 \Omega$ and $15 \Omega$, respectively. With the typical motional resistance of $R_{m} \sim 1 \mathrm{k} \Omega$ for a

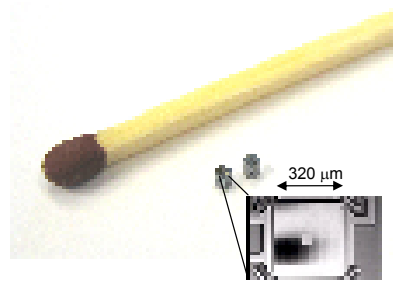

(a) Packaged resonators and a known of a microresonator. size scale. Inset: Microscope image

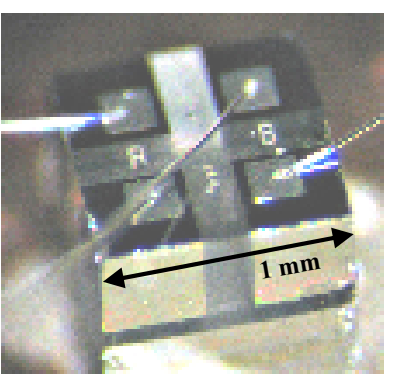

(b) Photo of a wire bonded resonator.
Fig. 1. Images of vacuum encapsulated resonators (size: $1 \mathrm{~mm}^{3}$ ). 


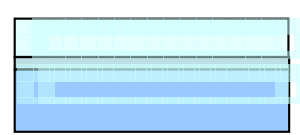

(a) Starting SOI wafer

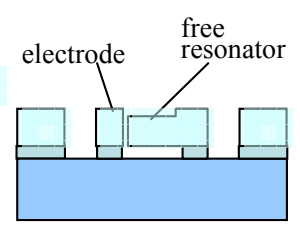

(c) Release etch of sacrificial oxide

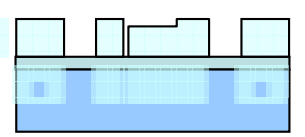

(b) Recessing and DRIE etching

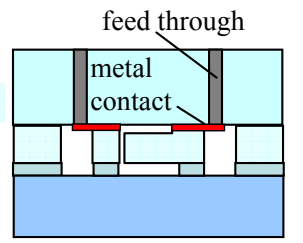
ing anodic bonding (d) Wafer encapsulation us-

Fig. 2. Fabrication process for wafer level vacuum encapsulated SOI resonators

microresonator, the feed through losses are negligible. In the future, thinning of the structure is planned to further reduce the package form factor.

\section{STUDIED RESONATORS}

The different resonators characterized are illustrated in Figure 3. The vibration of the square-extensional (se) resonator (Figure 3(a)) is characterized as plate expanding and contracting in-plane while preserving the square shape [8], [9]. The side length of the resonator corresponds to the half acoustic wavelength. To distinguish from the flexural vibration modes, the resonator is also referred to as a bulk acoustic wave (BAW) resonator. The electrical contact to the resonator is provided by the corner anchors. For added mechanical stability and to suppress low frequency vibrations, the resonator is also anchored from the center to the substrate.

The clamped-free (cf) beam resonator in Figure 3(b) vibrates in-plane and is anchored from the single point only. The length and width of the beam are $100 \mu \mathrm{m}$ and $4 \mu \mathrm{m}$, respectively.

The clamped-clamped (cc) beam resonator in Figure 3(c) is similar to the clamped-free resonator but it is anchored from two points. The length and width of the beam are $200 \mu \mathrm{m}$ and $4 \mu \mathrm{m}$, respectively.

The piston resonator in Figure 3(d) differs from the other investigates resonators as it vibrates out-of-plane. Thus, it has no side electrodes but the motion is excited and sensed with a metal electrode on the glass cover.

Figure 4 shows an example of measured resonator transmission curves that are used to extract the resonator quality factor and resonance frequency. Table I shows the summary of resonator properties in addition to the dc bias and ac excitation levels used in the stability characterization.

\section{MEASUREMENT SET-UP}

The measurement system was based on a HP 4396B network analyzer in transmission mode. The wire bonded encapsulated resonators (two samples of each resonator type) were mounted to a multi-channel measurement system that allowed a simultaneous monitoring of all the samples. The resonators

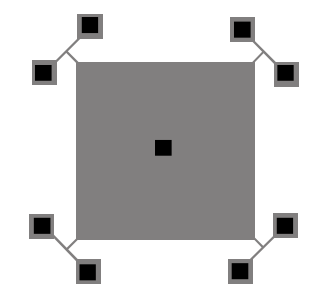

(a) Square-extensional (se) (b) Clamped-free (cf) beam resplate resonator. onator.

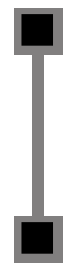

(c) Clamped-clamped (cc) beam resonator.

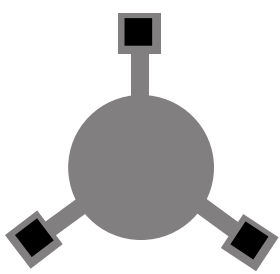

(d) Piston resonator.
Fig. 3. Schematic of the resonators characterized for stability. The black squares are the anchoring points to the substrate.

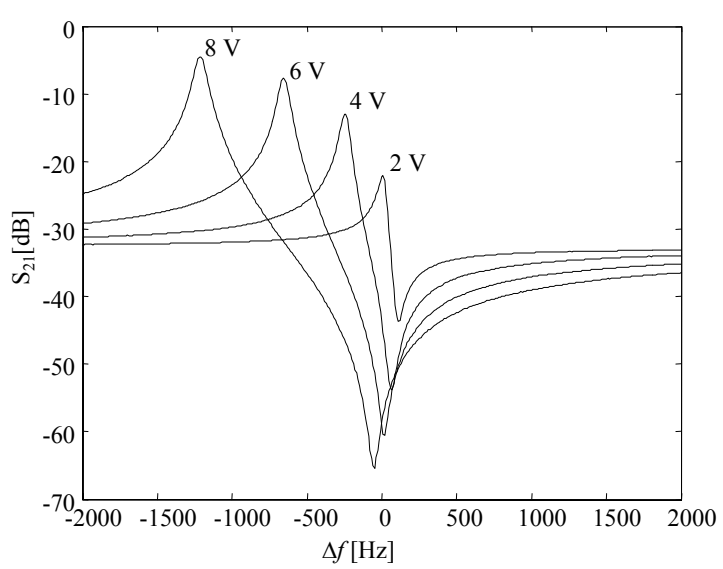

Fig. 4. Measured transmission curves for the piston resonator at different dc bias voltages.

TABLE I

RESONATOR CHARACTERISTICS AND TEST SET-UP

\begin{tabular}{lcccc}
\hline & $f_{0}$ & $Q$ & $U_{\text {bias }}$ & $u_{a c}$ \\
\cline { 2 - 5 } & $\mathrm{kHz}$ & & $\mathrm{V}$ & $\mathrm{dBm}$ \\
\hline se-plate & $13.0 \cdot 10^{3}$ & 100000 & 95 & -10 \\
cf-beam & 511 & 40000 & 20 & -25 \\
cc-beam & 782 & 30000 & 29 & -40 \\
piston & 671 & 5000 & 5 & -35 \\
\hline
\end{tabular}

were biased using an external bias voltage source and the bias voltage was constantly on. The transmission response of the resonators was recorded at 5-minute intervals. For measurement, the samples were installed into a steel container that was successively placed inside a climate chamber. The 
temperature inside the steel container was $30 \pm 0.1{ }^{\circ} \mathrm{C}$. As the resonators exhibit a sizeable temperature coefficient of $-28 \mathrm{ppm} / \mathrm{K}$, accurate temperature information is needed in order to decompose the temperature-induced variations from the recorded frequency data. For the measurement of the most stable devices (drift less than $10 \mathrm{ppm} / \mathrm{month}$ ), the device temperature was measured using Pt100 sensors which was placed in good thermal contact with the encapsulated devices. An Agilent 34970A Data Acquisition unit in four point resistance measurement mode was used to read out the temperature. For characterizing the less stable devices, the chamber temperature was recorded. The climate chamber was also used to subject the resonators to temperature cycling in order to determine the resonance frequency temperature coefficients and to test for possible temperature hysteresis effects.

\section{Measured stability}

Figure 5 shows the measured frequency drifts for the different types of resonators during one month. No changes in the resonator quality factors were observed during the measurement period indicating that the vacuum pressures inside the packages remained unchanged. The piston and clamped-clamped beam resonators show significant drifts of over $100 \mathrm{ppm} /$ week. Moreover, the drift varied significantly from sample to sample. This large drift is attributed to the packaging related stresses: Anchoring the devices from several points makes the resonant frequency sensitive to the substrate movements. A further indication of the packaging related stress effects is obtained by temperature cycling samples. The average frequency-temperature coefficients were -120 $\mathrm{ppm} / \mathrm{K}$ and $-50 \mathrm{ppm} / \mathrm{K}$ for the packaged cf-beam and piston resonators, respectively. This is substantially different from the frequency-temperature coefficient of $-28 \mathrm{ppm} / \mathrm{K}$ expected for the pure single-crystal silicon resonators. After the temperature cycles at $40{ }^{\circ} \mathrm{C}$ and $90{ }^{\circ} \mathrm{C}$, the resonant frequency of the clamped-clamped beam and piston resonators changed. Moreover, the slope of the drift curve changed form positive to negative. This may be explained by slow relaxation of the temperature induced stresses of the silicon-glass package.

The magnitude of the package induced stresses can be estimated from the frequency shift of the clamped-clamped beam. A first order approximation for the resonance frequency of a strained cc-beam is

$$
f=a_{1} \frac{w}{L^{2}} \sqrt{\frac{E}{\rho}} \sqrt{1+0.3 \frac{L^{2}}{w^{2}} S},
$$

where $a_{1}$ is a constant, $E$ is Young's modulus, $\rho$ is density, and $S$ is the axial strain [14]. Thus, the frequency shift due to change in strain $\Delta S$ is

$$
\Delta f=0.15 \frac{L^{2}}{w^{2}} \Delta S
$$

From Equation (2), we estimate that a strain of $10^{-6}$ or stress of $0.2 \mathrm{MPa}$ corresponds to $400 \mathrm{ppm}$ frequency shift. Stresses of this magnitude are typically encountered in packages showing the need for stress relieve structures if this type of resonators are used for reference applications.

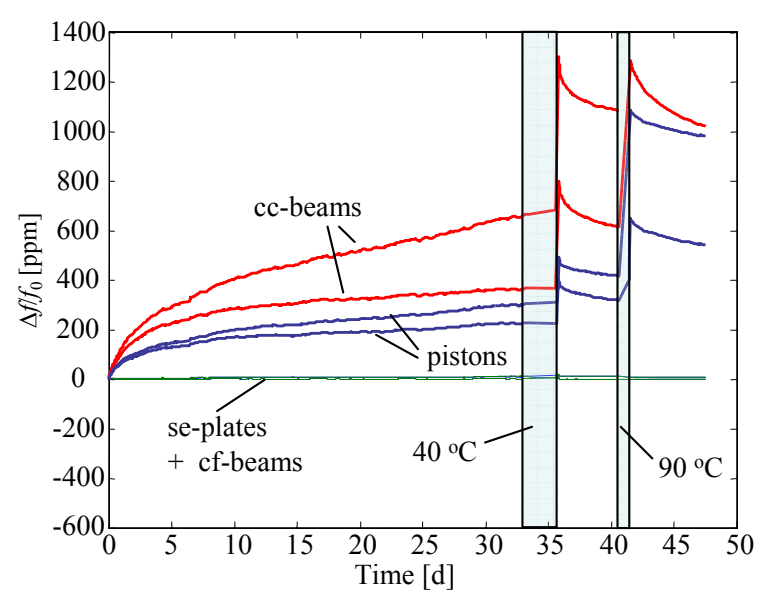

(a) Piston and cc-beam resonators exhibit large drifts and temperature hysteresis.

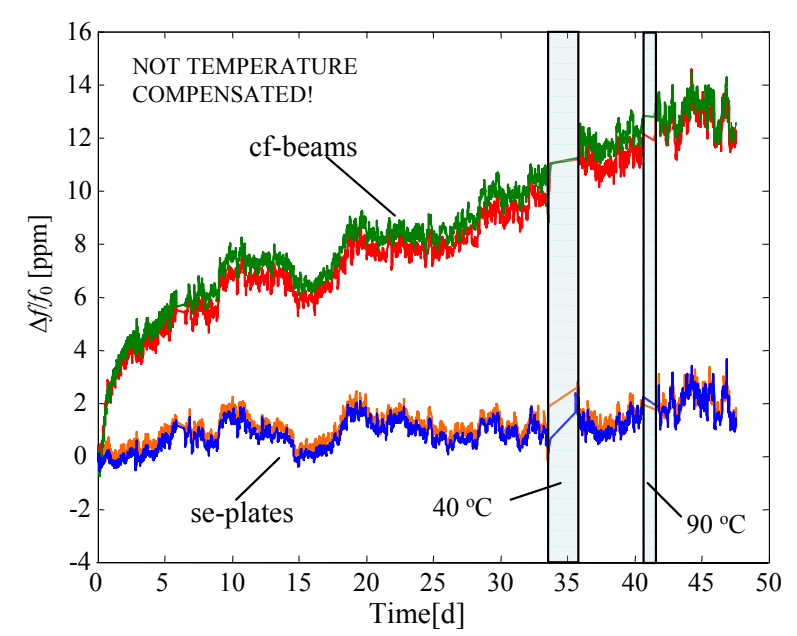

(b) Se-plate and cf-beam resonators demonstrate ppm-level stability. No temperature hysteresis is observed. The frequency fluctuations of the samples are tightly correlated indicating external disturbance (e.g. temperature) and is not indication of true drift.

Fig. 5. Measured drift for the four different resonator types. The shaded areas indicate cycling at elevated temperature to check for possible temperature hysteresis effects. Temperature variations have not been compensated.

Figure 5(b) shows a close-up of the stable squareextensional plate and clamped-free beam resonators. No temperature hysteresis is observed even for the temperature cycling at $90^{\circ} \mathrm{C}$. The cf-beam resonators that are anchored from a single point change less than $10 \mathrm{ppm}$ in one month. Even better performance, change about $1 \mathrm{ppm}$, is obtained with the square-extensional mode plate resonators. Moreover, the frequency shifts for the two measured se-plate samples are tightly correlated indicating that the shifts may be due to an external disturbance, e.g. temperature, and not true drift.

To accurately decompose the temperature induced variations, Pt100 temperature sensors was attached to the packaged devices. The results are shown in Figure 6 for the se-plates. Due to small temperature variations (Figure 6(b)), the measured frequency (Figure 6(a)) for the two samples fluctuates. The temperature compensated frequency (Figure 6(c)), how- 


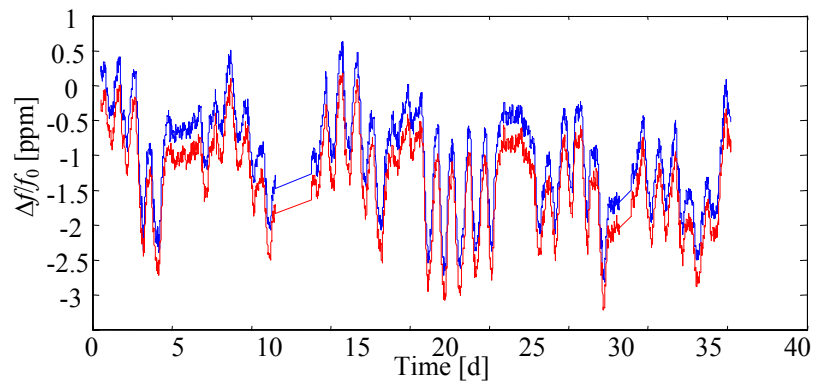

(a) Measured frequency shift for two se-plates.

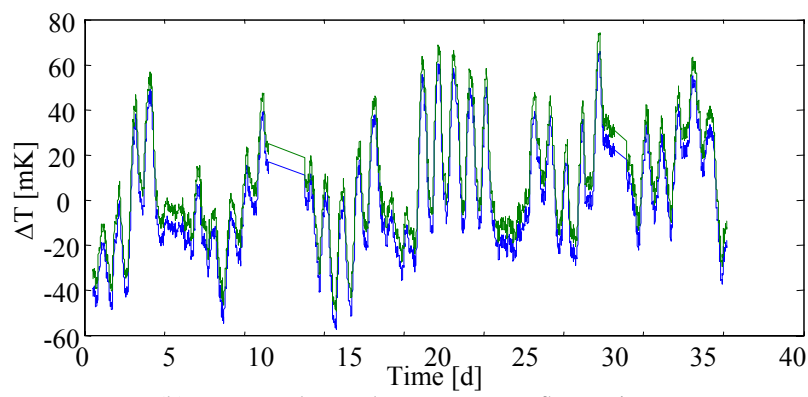

(b) Measured sample temperature fluctuations.

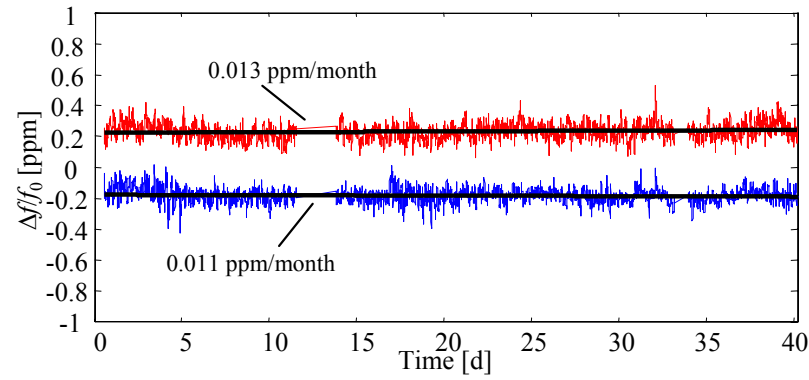

(c) Temperature compensated frequency shifts (drifts).

Fig. 6. Frequency shift for two se-plates over 40 days. From the temperature compensated frequency shift, a linear line regression gives drift of $\sim 0.01$ $\mathrm{ppm} / \mathrm{month}$.

ever, is very stable with linear line regression giving an average drifts of $0.013 \mathrm{ppm} / \mathrm{month}$ and $0.011 \mathrm{ppm} / \mathrm{month}$ for the two samples. Due to noise in the measurement, these very small numbers will have to be verified with longer measurements. However, it is clear that the drift is better than $0.1 \mathrm{ppm} / \mathrm{month}$. Similar measurement for the cf-beams resulted in drift of $4 \mathrm{ppm} / \mathrm{month}$.

\section{DisCUSSIONS}

Silicon resonators were vacuum encapsulated with a low cost wafer level packaging process and the stability was found to be suitable for reference applications. The plate resonators had the smallest drift $(<0.1 \mathrm{ppm} / \mathrm{month})$ and did not show any temperature hysteresis effects. The good performance of the plate resonators is attributed to the bulk acoustic nature of the vibration mode that makes the devices insensitive to the package stresses. The plate resonators also have a larger volume-to-surface ratio than flexural resonators making them less sensitive to the surface effects. These factors may explain why flexural clamped-free resonators showed orderof-magnitude larger drift of $4 \mathrm{ppm} / \mathrm{month}$.

The packaged clamped-clamped beam and piston resonators in this study had large drift and are unsuitable for reference applications. The drift was attributed to the package stresses. Different cover material e.g. silicon may reduce the stress. However, if low cost packaging techniques such as plastic molding and solder joining (flip-chip) are to be used for these stress sensitive components, it is expected that some form of stress relieve is required to decouple package stresses from the resonator.

In conclusion, we report the best stability performance hitherto obtained with microresonators. The achievement of sub-ppm level stability for wafer level encapsulated resonators is a significant milestone in realizing a commercially viable MEMS reference oscillator.

\section{ACKNOWLEDGMENT}

The financial support from VTI Technologies, Okmetic, and Tekes (National Technology Agency of Finland) is acknowledged.

\section{REFERENCES}

[1] J. Vig and T. Meeker, "The aging of bulk acoustic wave resonators, filters and oscillators," in Proceedings of the 45th Annual Symposium on Frequency Control, Los Angeles, CA, 29-31 May 1991, pp. 77-101.

[2] J. R. Vig and A. Ballato, Ultrasonic Instruments and Devices, E. P. Papadakis, Ed. San Diego, CA: Academic Press, 1999.

[3] B. Razavi, RF Microelectronics. Prentice Hall, 1998.

[4] T. Mattila, O. Jaakkola, J. Kiihamäki, J. Karttunen, T. Lamminmäki, P. Rantakari, A. Oja, H. Seppä, H. Kattelus, and I. Tittonen, "14 MHz micromechanical oscillator," Sensors and Actuators A, vol. 97-98, pp. 497-502, Apr. 2002

[5] W. T. Hsu and C. T. C. Nguyen, "Stiffness-compensated temperatureinsensitive micromechanical resonators," in IEEE Int. Micro Electro Mechanical Systems Conf, Las Vegas, Nevada, 20-24 Jan. 2002, pp. 731-734.

[6] T. Mattila, J. Kiihamäki, T. Lamminmäki, O. Jaakkola, P. Rantakari, A. Oja, H. Seppä, H. Kattelus, and I. Tittonen, "12 MHz micromechanical bulk acoustic mode oscillator," Sensors and Actuators A, vol. 101, no. 1-2, pp. 1-9, Sept. 2002.

7] V. Kaajakari, T. Mattila, A. Oja, and H. Seppä, "Nonlinear limits for single-crystal silicon microresonators," J. Microelectromech. Syst., vol. 13, no. 5, pp. 715-724, Oct. 2004.

[8] V. Kaajakari, T. Mattila, A. Oja, J. Kiihamäki, H. Kattelus, M. Koskenvuori, P. Rantakari, I. Tittonen, and H. Seppä, "Square-extensional mode single-crystal silicon micromechanical RF-resonator," in Transducers'03, The 12th International Conference on Solid-State Sensors and Actuators, Boston, MA, 8-12 Jun. 2004, pp. 951-954.

[9] V. Kaajakari, T. Mattila, A. Oja, J. Kiihamäki, and H. Seppä, "Squareextensional mode single-crystal silicon micromechanical resonator for low phase noise oscillator applications," IEEE Electron Device Lett., vol. 25, no. 4, pp. 173-175, Apr. 2004.

[10] Y.-W. Lin, S. Lee, S.-S. Li, Y. Xie, Z. Ren, and C. T.-C. Nguyen, "Seriesresonant VHF micromechanical resonator reference oscillators," IEEE J. Solid-State Circuits, vol. 39, no. 12, pp. 2477-2491, Dec. 2004

[11] J. A. Henry, Y. Wang, and M. A. Hines, "Controlling energy dissipation and stability of micromechanical silicon resonators with self-assembled monolayers," Applied Physics Letters, vol. 84, no. 10, pp. 1765-1767, Mar. 2004.

[12] M. Koskenvuori, T. Mattila, A. Häärä, J. Kiihamäki, I. Tittonen, A. Oja and H. Seppä, "Long-term stability of single-crystal silicon microresonators," Sensors and Actuators A, vol. 115, no. 1, pp. 23-27, Sept. 2004.

[13] VTI Technologies Oy, www.vti.fi.

[14] W. C. Young, Roark's Formulas for Stress and Strain, 6th ed. McGrawHill, 1989. 